\section{SERUM TRANSAMINASE ELEVATIONS IN INFANTS WITH ACUTE GASTROENTERITIS :IS IT HEPATIC HYPOFERFUSION OR ROTA VIRUS ASSOCIATED HEPATITIS?}

\author{
S.H. Barakat ${ }^{1}$, R. Harfoush ${ }^{2}$
}

${ }^{1}$ Pediatrics, Faculty of Medicine, Alexandria University, Alexandia, ${ }^{2}$ Microbiology, Faculty of Medicine, Alexandria University, Alexandria, Egypt

Objective: to examine the frequency of hepatic transaminase elevations in infants with acute gastroenteritis

Methods: Over a 8- weeks period, 35 of 130 infants admitted with acute gastroenteritis were found to have human rotavirus (HRV) gastroenteritis using stool antigen testing. Sera 130 infants were analyzed for alanine aminotransferase (ALT), aspartate aminotransferase, alkaline phosphatase, creatine phosphokinase (CPK), lactate dehydrogenase (LDH), total and direct bilirubin, and creatinine .

Results: Thirty four infants (26\%) had elevated ALT and AST. ALT elevations were 1-3 times the normal in $24(\sim 18 \%)$ and $6-20$ times the normal in $10(\sim 8 \%$ ) infants; the latter group showed the clinical and biochemical criteria of ischemic hepatopathy. In all infants transaminases normalized within 3-10 days. Transaminase elevations were not significantly associated with age, sex, duration of gastroenteritis or with any of the following symptoms; fever $(>38)$, vomiting or blood in the stools. A border line significant association was detected between HRV stool positivity and elevated ALT\&AST levels (40\% HRV positive VS. 21\% HRV negative odd ratio=2.5 95\% $\mathrm{Cl} 1-3.2 \mathrm{p}=0.05)$. Infant with severe dehydration had an estimated 31.5 fold higher odds of having elevated ALT \& AST compared with those no dehydration( $57,4 \%$ respectively).Multivariate logistic regression analysis, showed that severe dehydration is still significantly associated with elevated Transaminase levels.

Conclusion: our data suggest that liver injury during gastroenteritis in infants is quit frequent but always self limiting if the underlying perfusion disturbance caused by severe dehydration is corrected.

Rotavirus positivity was marginally associated with elevated transaminase levels.

\section{MONO-SYMPTOMATIC CYSTIC FIBROSIS (CF): A CASE REPORT}

\author{
J. Gonçalves ${ }^{1}$, F. Neiva ${ }^{1}$, H. Antunes ${ }^{1,2}$
}

${ }^{1}$ Pediatric Gastrenterology Unit, Braga Hospital,

${ }^{2}$ Life and Health Sciences Research Institute

(ICVS), School of Health Sciences, University of Minho, Braga, Portugal

Background: CF is a monogenic disorder with heterogeneous phenotypic presentation. In atypical cases, the sweat test may show border-line or normal values and the diagnosis involves the search for mutations in the CFTR gene, clinical features and follow-up.

Aim and clinical case: We present a case of an adolescent boy affected by recurrent acute pancreatitis (AP) started at puberty, with normal sweat values, carrier of compound heterozygosity (I507del+IVS8(5T)). Attending this result we performed other studies to exclude other possible CFTR-related signs, including respiratory infection and function or azoospermia. The chest radiography, spirometry, semen analysis and ultrasonography of the vas deferens and the seminal vesicles were normal. Tests for pancreatic endocrine and exocrine function were also normal. To complete the genetic evaluation, the siblings and both asymptomatic parents were tested because they are potentially at risk of similar disease. This genetic study showed that the father and the two siblings are carriers of the severe CFTR mutation (I507del) and the mother is carrier of the mutation IVS8 5 T.

Conclusion: The 1507del mutation is a severe CFTR mutation. In contrast, the IVS8(5T) mutation is not associated with clinical cystic fibrosis but, in compound heterozigosity with a more severe mutation, can be causative of mono- or oligosymptomatic CFTR-related disorders. This is probably the first case report of an atypical CF in a patient with the compound heterozygosity 1507del+IVS8 5T. It confirms the challenges and difficulties for diagnosing atypical CF and highlights the role of CFTR mutations in the pathogenesis of idiopathic pancreatitis. 